\title{
Controle genético e tolerância ao estresse de calor em populações híbridas e em cultivares de trigo(1)
}

\begin{abstract}
Moacil Alves de Souza(2) e Magno Antonio Patto Ramalho ${ }^{(3)}$
Resumo - O objetivo deste trabalho foi obter informações sobre o controle genético da produtividade de grãos de trigo sob condições de estresse de calor, o grau de tolerância ao estresse de calor de algumas cultivares e linhagens e a identificação de populações segregantes promissoras para a obtenção de linhagens adaptadas às condições da região central do Brasil. Foram avaliados 13 parentais e 40 populações provenientes de um dialelo parcial, nas gerações $F_{1}$ e $F_{2}$ em condições de campo, em Lavras e Patos de Minas, MG, com as semeaduras feitas no verão (fevereiro) e no inverno (maio). Houve diferença de tolerância ao calor entre os parentais e entre as populações híbridas quanto ao caráter produção de grãos, e ambos os efeitos aditivos e não-aditivos foram importantes no controle genético deste caráter na presença ou ausência de calor. Apesar da ocorrência de interações populações x épocas de semeadura, a alta repetibilidade dos efeitos da capacidade geral de combinação permite inferir a possibilidade de acelerar os programas de melhoramento na região, efetuando-se a seleção tanto na época de verão quanto no inverno.
\end{abstract}

Termos para indexação: Triticum aestivum, cruzamento dialélico, métodos de melhoramento.

\section{Genetic control and tolerance to the heat stress in wheat hybrid population and cultivars}

\begin{abstract}
The objective of this work was to get information about the genetic control of the grain yield under heat stress conditions, the tolerance of cultivar and the identification of promising segregation populations for selections of lines adapted to the conditions of the central region of Brazil. Thirteen parents and 40 populations in $\mathrm{F}_{1}$ and $\mathrm{F}_{2}$ generations derived by partial diallel crosses were evaluated. The experiments were conducted under field conditions in Lavras and Patos de Minas, MG, Brazil, sowing the seeds in the summertime (February) and in the winter (May). There was difference of heat tolerance among parents and hybrid populations for grain yield character, and both additive and non-additive effects were important in the genetic control of grain yield character in the presence or absence of heat. In spite of the occurrence of populations x sowing time interactions, the high repeatability of the effects of the general combining ability suggests the possibility of the selection in the summertime and winter facilitating to advance the breeding programs in the region.
\end{abstract}

Index terms: Triticum aestivum, diallel crossing, breeding methods.

\section{Introdução}

A principal limitação da cultura do trigo na região central do Brasil é a ocorrência de temperaturas muito elevadas durante o ciclo. O excesso de calor afeta vários caracteres das plantas, e, como

(1) Aceito para publicação em 2 de novembro de 2000 Extraído da Tese de Doutorado apresentada pelo primeiro autor à Universidade Federal de Lavras (Ufla), Lavras, MG

(2) Universidade Federal de Viçosa, Dep. de Fitoctenia, CEP 36571-000 Viçosa, MG. E-mail: moacil@mail.ufv.br

(3)Ufla, Dep. de Biologia, Caixa Postal 32, CEP 37200-000 Lavras, MG. E-mail: magnoapr@ufla.br conseqüência, a produtividade de grãos. Esta limitação restringe a expansão da cultura para regiões com altitudes inferiores a $1.000 \mathrm{~m}$, independentemente do sistema de cultivo, ou seja, com irrigação ou em regime de sequeiro. O cultivo dessa gramínea é uma opção para a rotação de culturas, necessária para manter as produtividades elevadas nas culturas de verão.

O sucesso da cultura do trigo na região central do Brasil, e especialmente no Estado de Minas Gerais, depende de vários fatores, dos quais a existência de cultivares tolerantes ao calor assume posição de destaque. $\mathrm{O}$ uso de cultivares adaptadas possibilita 
que o trigo seja cultivado em regiões de clima do tipo Mediterrâneo e outras regiões tropicais. Em Minas Gerais, há, ainda, a possibilidade de semeadura no final do verão, quando ainda existe disponibilidade de chuvas, o que poderia contribuir para a redução do custo de produção da lavoura de trigo. Neste caso, o excesso de calor, associado às deficiências hídricas periódicas, constitui o principal fator de redução no potencial de produtividade da cultura do trigo.

Existe variabilidade quanto à tolerância ao calor, que tem sido constatada em diversos trabalhos (Shpiler \& Blum, 1986; Moffatt et al., 1990; Acevedo et al., 1991); contudo, nas condições brasileiras, esse tipo de trabalho é pouco freqüente. A maioria dos resultados sobre a genética da tolerância ao calor é obtida em condições controladas, utilizando caracteres fisiológicos (Porter et al., 1995; Fokar et al., 1998). Os resultados destes trabalhos devem ser utilizados em complementação às pesquisas envolvendo caracteres agronômicos, visto que as condições em que são realizados não retratam o que de fato ocorre no campo (Acevedo et al., 1991).

A obtenção de informações sobre o controle genético da tolerância ao calor é uma condição para tornar mais eficientes os programas de melhoramento a serem conduzidos.

O objetivo deste trabalho foi obter informações sobre o controle genético da produtividade de grãos de trigo sob condições de estresse de calor, o grau de tolerância ao estresse de calor de algumas cultivares e linhagens e a identificação de populações segregantes promissoras para obtenção de linhagens adaptadas às condições da região central do Brasil.

\section{Material e Métodos}

Os experimentos foram conduzidos em Lavras, MG, e em Patos de Minas, MG, no período de 1997 a 1999, utilizando-se 13 genitores, que foram escolhidos e estratificados em dois grupos, conforme suas aptidões de cultivo. O grupo I foi constituído por cinco cultivares utilizadas em lavouras, e três linhagens em fase de avaliação em cultivo com irrigação, todos de porte baixo. No grupo II foram incluídas três cultivares recomendadas aos agricultores, e duas linhagens em fase de testes no cultivo de sequeiro, predominando porte médio a alto. Estes genitores foram cruzados no esquema de dialelo parcial, em condições de campo, obtendo-se 40 populações híbridas.
Foram utilizadas duas épocas de semeadura, nos locais mencionados. A primeira época de semeadura, denominada semeadura de verão, foi realizada no mês de fevereiro, onde predominam temperaturas elevadas durante 0 ciclo de desenvolvimento da cultura (Tabelas 1 e 2). A segunda época foi efetuada no final do mês de abril e meados do mês de maio (inverno), período a partir do qual ocorrem temperaturas mais amenas durante a maior parte do ciclo da cultura.

As populações híbridas e os genitores foram avaliados utilizando-se o delineamento em látice ou em blocos casualizados, com duas ou três repetições. Na geração $F_{1}$, cada parcela foi constituída de uma linha de $3,0 \mathrm{~m}$ de comprimento, no espaçamento de $40 \mathrm{~cm}$ entre linhas e $15 \mathrm{~cm}$ entre plantas. Na geração $F_{2}$, as parcelas foram constituídas de quatro linhas de $3,0 \mathrm{~m}$, no espaçamento de $40 \mathrm{~cm}$ entre linhas, utilizando-se 50 plantas por metro linear.

Foram adotadas práticas culturais uniformes em todos os experimentos, para minimizar a influência de fatores bióticos e abióticos na expressão dos tratamentos. Os experimentos receberam $500 \mathrm{~kg} /$ ha da fórmula 4-14-8, por ocasião da semeadura, e $200 \mathrm{~kg} / \mathrm{ha}$ de sulfato de amônio aplicado em cobertura, no início do perfilhamento $(100 \mathrm{~kg} / \mathrm{ha})$, e no estádio de diferenciação floral (100 kg/ha). O suprimento de água no solo foi mantido por irrigações, que em períodos de ausência de chuvas impôs um turno de rega semanal com lâmina de 20 a $25 \mathrm{~mm}$. Em todas as situações, foram adotadas as recomendações existentes para a cultura no controle das plantas daninhas, de doenças e de pragas (Comissão CentroBrasileira de Pesquisa de Trigo, 1999).

Os dados de produtividade de grãos foram submetidos a análise de variância dialélica, utilizando-se o modelo 1 , método IV, proposto por Griffing (1956), adaptado a dialelos parciais, conforme apresentado por Cruz \& Regazzi (1994). Utilizando-se dos valores da capacidade geral de combinação (CGC) dos parentais, foi calculada a repetibilidade destas estimativas, nos quatro ambientes, locais e épocas de semeadura, para a geração $F_{1}$ e $F_{2}$. A estimativa da repetibilidade $(Z)$ foi obtida por meio da esperança do quadrado médio da análise de variância dos valores da CGC, segundo a expressão: $\mathrm{Z}=\left(\mathrm{QM}_{\text {Pais }}-\mathrm{QM}_{\mathrm{E}}\right) / \mathrm{QM}_{\text {Pais}}$, em que: $\mathrm{QM}_{\text {Pais }}$ é o quadrado médio dos efeitos de pais; $\mathrm{QM}_{\mathrm{E}}$ é o quadrado médio do erro da referida análise.

A quantificação dos efeitos de estresse de calor ocorrido no verão em relação ao inverno foi obtida pela porcentagem de redução $(\% \mathrm{R})$ da produtividade de grãos observada nas duas épocas, em cada local, conforme a expressão (Wardlaw et al., 1989): \% $\mathrm{R}=\left[1-\left(\mathrm{P}_{\mathrm{V}} / \mathrm{P}_{\mathrm{I}}\right)\right] * 100$, cujo erro-padrão foi estimado por $\operatorname{EP}(\% \mathrm{R})=[\mathrm{V}(\% \mathrm{R})]^{1 / 2}$. 
Tabela 1. Porcentagem de ocorrências de faixas de temperatura $\left({ }^{\circ} \mathrm{C}\right)$ e médias das temperaturas médias, máximas e mínimas por fases de desenvolvimento médio das plantas de trigo. Ano de $1997^{(1)}$.

\begin{tabular}{|c|c|c|c|c|c|c|c|}
\hline \multirow[t]{2}{*}{ Fase do ciclo } & \multicolumn{4}{|c|}{ Intervalos de temperatura $\left({ }^{\circ} \mathrm{C}\right)$ em $\%$} & \multicolumn{3}{|c|}{ Temperatura $\left({ }^{\circ} \mathrm{C}\right)$} \\
\hline & $t>25$ & $20<\mathrm{t} \leq 25$ & $15<\mathrm{t} \leq 20$ & $\mathrm{t}<15$ & Média & Máxima & Mínima \\
\hline & \multicolumn{7}{|c|}{ Lavras - Verão/97 } \\
\hline Emergência/Perfilhamento & 18,33 & 34,17 & 47,00 & 0,15 & 21,65 & 28,42 & 17,01 \\
\hline Perfilhamento/Espigamento & 4,03 & 33,89 & 52,92 & 9,17 & 19,73 & 25,92 & 15,36 \\
\hline Espigamento/Maturação & 1,17 & 18,86 & 37,21 & 42,76 & 17,66 & 24,77 & 12,90 \\
\hline \multirow[t]{2}{*}{ Todo ciclo } & 5,77 & 26,30 & 43,60 & 21,20 & 19,18 & 25,97 & 14,54 \\
\hline & \multicolumn{7}{|c|}{ Lavras - Inverno/97 } \\
\hline Emergência/Perfilhamento & 1,83 & 14,33 & 32,67 & 50,67 & 16,24 & 24,00 & 10,80 \\
\hline Perfilhamento/Espigamento & - & - & - & - & 17,67 & 26,60 & 11,28 \\
\hline Espigamento/Maturação & - & - & - & - & 20,21 & 29,27 & 13,29 \\
\hline \multirow[t]{2}{*}{ Todo ciclo } & - & - & - & - & 18,46 & 27,19 & 12,04 \\
\hline & \multicolumn{7}{|c|}{ Patos de Minas - Verão/97 } \\
\hline Emergência/Perfilhamento & 23,83 & 40,83 & 35,33 & 0,00 & 22,39 & 28,45 & 18,61 \\
\hline Perfilhamento/Espigamento & 13,44 & 30,24 & 52,42 & 3,90 & 20,59 & 26,42 & 16,65 \\
\hline Espigamento/Maturação & 8,05 & 28,79 & 51,61 & 11,55 & 19,52 & 25,77 & 14,44 \\
\hline \multirow[t]{2}{*}{ Todo ciclo } & 13,67 & 32,25 & 47,79 & 6,29 & 20,30 & 26,59 & 16,08 \\
\hline & \multicolumn{7}{|c|}{ Patos de Minas - Inverno/97 } \\
\hline Emergência/Perfilhamento & 5,17 & 23,50 & 47,83 & 23,50 & 17,90 & 24,75 & 13,05 \\
\hline Perfilhamento/Espigamento & 7,74 & 29,29 & 42,62 & 20,36 & 18,84 & 26,46 & 13,01 \\
\hline Espigamento/Maturação & 31,97 & 29,06 & 30,15 & 8,81 & 22,27 & 30,58 & 15,49 \\
\hline Todo ciclo & 17,61 & 27,79 & 38,52 & 16,08 & 20,12 & 27,88 & 14,10 \\
\hline
\end{tabular}

${ }^{(1)}$ Dados climáticos obtidos nos postos meteorológicos de Lavras, MG (altitude: $918,87 \mathrm{~m}$; latitude: $21^{\circ} 14^{\prime}$ S; longitude: $45^{\circ} 0^{\prime} \mathrm{W}$ ) e Patos de Minas, MG (altitude: $940,28 \mathrm{~m}$; latitude: $18^{\circ} 36^{\prime} \mathrm{S}$; longitude: $46^{\circ} 31^{\prime} \mathrm{W}$ ), próximo à área experimental (300 m), vinculados ao 5o Distrito de Meteorologia do Ministério da Agricultura e Reforma Agrária, em Belo Horizonte, MG.

Tabela 2. Porcentagem de ocorrências de faixas de temperatura $\left({ }^{\circ} \mathrm{C}\right)$ e médias das temperaturas médias, máximas e mínimas por fases de desenvolvimento médio das plantas de trigo. Ano 1998/1999(1).

\begin{tabular}{|c|c|c|c|c|c|c|c|}
\hline \multirow[t]{2}{*}{ Fase do ciclo } & \multicolumn{4}{|c|}{ Intervalos de temperatura $\left({ }^{\circ} \mathrm{C}\right)$ em $\%$} & \multicolumn{3}{|c|}{ Temperatura $\left({ }^{\circ} \mathrm{C}\right)$} \\
\hline & $\mathrm{t}>25$ & $20<\mathrm{t} \leq 25$ & $15<\mathrm{t} \leq 20$ & $\mathrm{t}<15$ & Média & Máxima & Mínima \\
\hline & \multicolumn{7}{|c|}{ Lavras - Verão/98 } \\
\hline Emergência/Perfilhamento & 30,00 & 35,17 & 34,83 & 0,00 & 23,64 & 20,30 & 18,84 \\
\hline Perfilhamento/Espigamento & 19,00 & 34,67 & 43,65 & 4,15 & 22,31 & 28,55 & 18,25 \\
\hline Espigamento/Maturação & 18,67 & 28,86 & 37,05 & 15,43 & 20,05 & 26,82 & 15,03 \\
\hline \multirow[t]{2}{*}{ Todo ciclo } & 21,38 & 32,09 & 38,22 & 8,31 & 21,47 & 28,10 & 16,71 \\
\hline & \multicolumn{7}{|c|}{ Lavras - Inverno/98 } \\
\hline Emergência/Perfilhamento & 2,00 & 19,17 & 39,50 & 39,33 & 16,64 & 23,27 & 12,00 \\
\hline Perfilhamento/Espigamento & 3,92 & 21,08 & 31,74 & 43,26 & 16,51 & 24,09 & 10,89 \\
\hline Espigamento/Maturação & 20,08 & 22,67 & 37,42 & 20,83 & 19,59 & 27,24 & 13,79 \\
\hline \multirow[t]{2}{*}{ Todo ciclo } & 10,43 & 21,37 & 36,12 & 32,07 & 17,94 & 25,34 & 12,46 \\
\hline & \multicolumn{7}{|c|}{ Patos de Minas - Verão/99 } \\
\hline Emergência/Perfilhamento & 20,16 & 42,67 & 37,17 & 0,00 & 22,07 & 28,01 & 18,53 \\
\hline Perfilhamento/Espigamento & 29,37 & 42,71 & 27,92 & 0,00 & 22,69 & 29,16 & 18,39 \\
\hline Espigamento/Maturação & 23,63 & 30,64 & 40,09 & 6,45 & 20,78 & 28,40 & 15,11 \\
\hline \multirow[t]{2}{*}{ Todo ciclo } & 10,43 & 36,61 & 36,61 & 3,26 & 21,52 & 28,46 & 16,70 \\
\hline & \multicolumn{7}{|c|}{ Patos de Minas - Inverno/99 } \\
\hline Emergência/Perfilhamento & 12,67 & 26,67 & 42,00 & 18,66 & 20,06 & 28,38 & 13,81 \\
\hline Perfilhamento/Espigamento & 19,56 & 25,46 & 37,85 & 17,13 & 19,51 & 27,48 & 13,22 \\
\hline Espigamento/Maturação & 19,53 & 28,75 & 41,23 & 10,49 & 19,98 & 27,43 & 14,19 \\
\hline Todo ciclo & 17,93 & 27,16 & 40,27 & 14,63 & 19,85 & 27,66 & 13,79 \\
\hline
\end{tabular}

${ }^{(1)}$ Dados climáticos obtidos nos postos meteorológicos de Lavras, MG (altitude: $918,87 \mathrm{~m}$; latitude: $21^{\circ}$ 14' S; longitude: $45^{\circ} 0^{\prime} \mathrm{W}$ ) e Patos de Minas, MG (altitude: $940,28 \mathrm{~m}$; latitude: $18^{\circ} 36^{\prime} \mathrm{S}$; longitude: $46^{\circ} 31^{\prime} \mathrm{W}$ ), próximos à área experimental $(300 \mathrm{~m})$, vinculados ao 5 o Distrito de Meteorologia do Ministério da Agricultura e Reforma Agrária, em Belo Horizonte, MG. 
A variância da \%R foi obtida conforme Wricke \& Weber (1986): $\mathrm{V}(\% \mathrm{R})=10^{4} *\left\{\left[\mathrm{~V}\left(\mathrm{P}_{\mathrm{V}}\right) / \overline{\mathrm{P}}_{\mathrm{I}}^{2}\right]+\left[\overline{\mathrm{P}}_{\mathrm{V}}^{2} * \mathrm{~V}\left(\mathrm{P}_{\mathrm{I}}\right) / \overline{\mathrm{P}}_{\mathrm{I}}^{4}\right.\right.$, em que: $\mathrm{V}\left(\mathrm{P}_{\mathrm{V}}\right) \mathrm{e} \mathrm{V}\left(\mathrm{P}_{\mathrm{I}}\right)$ são as variâncias do erro nos experimentos de verão e inverno, respectivamente; $\overline{\mathrm{P}}_{\mathrm{V}}$ e $\overline{\mathrm{P}}_{\mathrm{I}}$ são as médias da produtividade de grãos nos experimentos de verão e inverno, respectivamente.

\section{Resultados e Discussão}

Na semeadura de verão, especialmente até a fase de perfilhamento, as plantas de trigo foram submetidas a condições bem adversas quanto às temperaturas (Tabelas 1 e 2). Observou-se reduzida germinação na semeadura de verão, quando se utilizaram sementes $F_{1}$, o mesmo não ocorrendo com as sementes $F_{2}$ no ano seguinte, nas mesmas condições. Todas as evidências apontam para a excessiva temperatura do solo na fase de germinação e emergência das plântulas e para o menor conteúdo de endosperma das sementes $F_{1}$, que normalmente tem o enchimento de grãos prejudicado pelo estresse do processo de hibridação. As temperaturas do solo, tomadas no posto meteorológico próximo à área experimental, em Lavras, e nos horários de $9 \mathrm{~h}, 15 \mathrm{~h}$ e $21 \mathrm{~h}$ foram de 23,30 e $27,7^{\circ} \mathrm{C}$, respectivamente, na fase de germinação no verão. Entretanto, no inverno do mesmo ano, as temperaturas foram de $13,7,20,9$ e $16,2^{\circ} \mathrm{C}$, com diferença de $10^{\circ} \mathrm{C}$ entre as duas épocas (Tabela 1). De acordo com Blum \& Sinmena (1994), durante o processo de germinação, toda fonte de C é dependente do estoque deste elemento no endosperma da semente, e, quando as temperaturas são elevadas, boa parte do $\mathrm{C}$ é perdida pela respiração. Esta constatação indica que a utilização de sementes $F_{1}$ em estudos de tolerância ao calor no campo deve ser evitada, dando-se preferência às sementes $F_{2}$.

As diferenças acentuadas de temperaturas entre as épocas de semeaduras contribuíram para que ocorresse diferença expressiva no desempenho das cultivares e das populações. As produções médias de grãos das populações $F_{2}$ em Lavras foram de $1.032,3 \mathrm{~g}$ por parcela no verão contra $2.308,0 \mathrm{~g}$ no inverno, ou seja, ocorreu redução de 55,3\% (Tabela 3$)$.

Um questionamento que poderia ser feito é que as diferenças em produtividade de grãos entre as duas épocas de semeadura pudessem estar influenciadas também pelos efeitos do fotoperíodo. De fato, em fevereiro, o comprimento do dia é de 13 horas, e em maio é de 11 horas, ou seja, ocorre uma diferença de duas horas entre as épocas de semeadura. Houve diferenças entre os genitores, mas entre as épocas de semeadura, verão e inverno, os valores da soma térmica são praticamente os mesmos, e há acentuada diferença no ciclo, indicando que a variação observada no ciclo dos parentais foi determinada predominantemente pelos efeitos de temperatura (Tabela 4). Admitindo-se a possível ausência dos efeitos do fotoperíodo sobre o comportamento dos genitores, pode-se pressupor que as diferenças de produtividades nas duas épocas devem ser resultantes dos efeitos das altas temperaturas ocorrentes no verão.

$\mathrm{Na}$ análise de variância dialélica, as interações dos efeitos das capacidades de combinação foram altamente significativas, e por isso, optou-se por apresentar as análises de variância do dialelo por ambientes (Tabela 3 ). Não houve boa concordância dos resultados das gerações $F_{1}$ e $F_{2}$. No caso da capacidade geral de combinação (CGC) do grupo I, na geração $\mathrm{F}_{1}$, foram constatadas diferenças significativas em todos os ambientes, ao passo que na geração $F_{2}$ isso ocorreu apenas nos experimentos de Patos de Minas. Quando se considerou o grupo II, diferenças significativas na geração $F_{1}$ só foram constatadas na semeadura de inverno, enquanto na $\mathrm{F}_{2}$ a $\mathrm{CGC}$ foi significativa em todos os ambientes.

A quantificação dos efeitos aditivos e não-aditivos, medida pelo coeficiente de determinação $\left(\mathrm{R}^{2}\right)$, indicou que a contribuição da capacidade específica de combinação (CEC) na geração $F_{1}$ foi marcante na variação total das populações, independentemente dos ambientes $\left(0,53 \leq R^{2} \leq 0,64\right)$. Na geração $F_{2}$, constatou-se que a maior contribuição para a variação das populações deveu-se a CGC II. Apesar de a CEC ter sido menor do que na $\mathrm{F}_{1}$, ainda superou a CGC I, ou seja, a participação dos efeitos não-aditivos continuou importante no controle do caráter produção de grãos na geração $\mathrm{F}_{2}$.

Como era esperado, na geração $\mathrm{F}_{1}$, dada a maior freqüência de locos em heterozigose, a contribuição da CEC foi maior, o que indica a presença de efeitos não-aditivos no controle da produção. Outra explicação para a maior contribuição dos efeitos nãoaditivos é a menor competição entre plantas na geração $\mathrm{F}_{1}$, em face da menor densidade de semeadura. 
$\mathrm{Na}$ literatura, existem vários relatos da maior expressividade dos efeitos não-aditivos em condições que favorecem o maior potencial de produção da planta, como, por exemplo, maior espaçamento entre plantas e uso de irrigação (Cregan \& Busch, 1978; Souza, 1993).

O resultado que comprova a existência de dominância é o fato de que em todos os casos o contraste pais vs. populações híbridas foi significativo, o que indica a presença de heterose, ou seja, de efeitos não-aditivos no controle do caráter produtividade de grãos. Borghi et al. (1989) relataram que as estimativas de vigor híbrido podem atingir 15\% acima da média dos pais em condições normais de semeadura em lavoura. A heterose é um fenômeno importante para a produtividade na cultura do trigo,

Tabela 3. Resumo da análise de variância dialélica da produção de grãos de trigo ${ }^{(1)}$.

\begin{tabular}{|c|c|c|c|c|c|}
\hline \multirow[t]{2}{*}{ Fonte de variação } & \multirow[t]{2}{*}{ GL } & \multicolumn{2}{|c|}{ Lavras } & \multicolumn{2}{|c|}{ Patos de Minas } \\
\hline & & Verão & Inverno & Verão & Inverno \\
\hline & & \multicolumn{4}{|c|}{ Geração $F_{1}(\mathrm{~g} / 20$ plantas $)$} \\
\hline Populações & 39 & $1.318,64^{\mathrm{ns}}$ & $8.203,50 * *$ & $1.671,26^{\mathrm{ns}}$ & $3.989,32 * *$ \\
\hline CGC I & 7 & $3.349,78^{*}$ & $4.338,40 * *$ & $3.797,76^{*}$ & $6.124,99 * *$ \\
\hline CGC II & 4 & $1.18,45^{\mathrm{ns}}$ & $20.836,97 * *$ & $720,36^{\mathrm{ns}}$ & $7.406,14 * *$ \\
\hline $\mathrm{CEC}$ & 28 & $9.82,31^{\mathrm{ns}}$ & $7.364,99 * *$ & $1.275,47^{\mathrm{ns}}$ & $2.967,28 * *$ \\
\hline Média (g/20 plantas) & 39 & 257,6 & 660,2 & 395,3 & 470,7 \\
\hline $\mathrm{R}^{2}(\mathrm{CGCI})$ & 7 & 0,456 & 0,095 & 0,408 & 0,276 \\
\hline $\mathrm{R}^{2}$ (CGCII) & 4 & 0,009 & 0,261 & 0,044 & 0,190 \\
\hline \multirow[t]{2}{*}{$\mathrm{R}^{2}(\mathrm{CEC})$} & 28 & 0,535 & 0,644 & 0,548 & 0,534 \\
\hline & & \multicolumn{4}{|c|}{ Geração $F_{2}(g / 4,8$ plantas $)$} \\
\hline Populações & & $17.596,62 * *$ & $39.541,23 * *$ & $49.640,56^{* *}$ & $39.448,52 * *$ \\
\hline CGC I & & $18.720,80^{\mathrm{ns}}$ & $12.042,76^{\mathrm{ns}}$ & $75.430,34 * *$ & $59.197,03 * *$ \\
\hline CGC II & & $66.312,25 * *$ & $171.393,93 * *$ & $275.950,90 * *$ & $162.356,04 * *$ \\
\hline $\mathrm{CEC}$ & & $10.356,20^{\mathrm{ns}}$ & $27.454,39 * *$ & $10.863,07^{\mathrm{ns}}$ & $16.953,18 *$ \\
\hline Média $\left(\mathrm{g} / 4,8 \mathrm{~m}^{2}\right)$ & & $1.032,27$ & $2.308,03$ & $1.070,27$ & $2.139,70$ \\
\hline $\mathrm{R}^{2}(\mathrm{CGC} \mathrm{I})$ & & 0,191 & 0,055 & 0,273 & 0,269 \\
\hline $\mathrm{R}^{2}$ (CGC II) & & 0,386 & 0,445 & 0,570 & 0,422 \\
\hline $\mathrm{R}^{2}(\mathrm{CEC})$ & & 0,422 & 0,500 & 0,157 & 0,308 \\
\hline
\end{tabular}

${ }^{(1)}$ CGC I: capacidade geral de combinação dos genitores do grupo I; CGC II: capacidade geral de combinação dos genitores do grupo II; CEC: capacidade específica de combinação; $\mathrm{R}^{2}$ : razão entre a soma de quadrados das fontes de variação CGC I ou CGC II ou CEC e a de populações. ns Não-significativo. $* \mathrm{e} * *$ Significativo a $5 \%$ e a $1 \%$ de probabilidade, respectivamente, pelo teste $\mathrm{F}$.

Tabela 4. Soma térmica $\left({ }^{\circ} \mathrm{C}\right.$.dia) e ciclo (dias) da emergência ao estádio de antese dos genitores de trigo envolvidos no cruzamento dialelo, em Lavras, MG.

\begin{tabular}{|c|c|c|c|c|c|c|c|c|}
\hline \multirow[t]{3}{*}{ Genitores } & \multicolumn{4}{|c|}{1997} & \multicolumn{4}{|c|}{1998} \\
\hline & \multicolumn{2}{|c|}{ Verão } & \multicolumn{2}{|c|}{ Inverno } & \multicolumn{2}{|c|}{ Verão } & \multicolumn{2}{|c|}{ Inverno } \\
\hline & ${ }^{\circ} \mathrm{C} . d i a$ & Ciclo & ${ }^{\circ} \mathrm{C}$.dia & Ciclo & ${ }^{\circ} \mathrm{C}$. dia & Ciclo & ${ }^{\circ}$ C.dia & Ciclo \\
\hline Anahuac & 1.213 & 59 & 1.073 & 63 & 1.216 & 54 & 1.137 & 68 \\
\hline BR 12 & 1.075 & 52 & 1.132 & 66 & 991 & 43 & 1.013 & 61 \\
\hline BR 26 & 1.233 & 60 & 1.036 & 61 & 1.058 & 46 & 1.104 & 66 \\
\hline CPAC 9186 & 1.233 & 60 & 1.151 & 67 & 1.193 & 53 & 1.174 & 70 \\
\hline EMBRAPA 22 & 1.095 & 53 & 1.132 & 66 & 945 & 41 & 974 & 59 \\
\hline EP 9320 & 994 & 48 & 1.132 & 66 & 969 & 42 & 1.034 & 62 \\
\hline IAC 24 & 1.034 & 50 & 1.113 & 65 & 1.058 & 46 & 1.013 & 61 \\
\hline IVI 931036 & 933 & 45 & 1.113 & 65 & 969 & 42 & 994 & 60 \\
\hline BH 1146 & 975 & 47 & 1.002 & 59 & 1.037 & 45 & 937 & 57 \\
\hline BR 24 & 1.132 & 55 & 1.054 & 62 & 1.193 & 53 & 1.013 & 61 \\
\hline EMBRAPA 21 & 1.213 & 59 & 1.191 & 69 & 1.111 & 49 & 1.137 & 68 \\
\hline EP 9287 & 1.014 & 49 & 1.151 & 67 & 1.058 & 46 & 1.053 & 63 \\
\hline EP 93541 & 1.056 & 51 & 1.113 & 65 & 1.014 & 44 & 937 & 57 \\
\hline Média & 1.092 & 53 & 1.107 & 65 & 1.062 & 46 & 1.040 & 63 \\
\hline
\end{tabular}


embora o controle genético deste caráter em trigo tenha indicado a predominância dos efeitos aditivos.

Houve diferenças entre as estimativas dos efeitos da capacidade geral de combinação dos genitores do grupo I $\left(\mathrm{g}_{\mathrm{i}}\right)$ e do grupo II $\left(\mathrm{g}_{\mathrm{j}}\right)$, tanto no verão quanto no inverno (Tabela 5). Os parentais que apresentaram os maiores valores da CGC em todos os ambientes foram CPAC 9186, EP 9320, EP 93541 e BR 24, enquanto no verão sobressaíram BH 1146 e Anahuac. Foi verificado que nem todos os parentais que apresentaram alta CGC tiveram um bom desempenho per se no referente à produtividade de grãos. Isto pode ser explicado pela constatação dos efeitos não-aditivos, significando a existência de certas combinações híbridas superiores para determinados genitores.

A despeito das interações detectadas entre a CGC e os ambientes, a estimativa da repetibilidade dos efeitos da CGC dos genitores do grupo I foi de $60,7 \%$, e para o grupo II foi de $88,0 \%$ (Tabela 5). Assim, pode-se inferir que o desempenho dos parentais quanto a CGC pode ser predito com boa margem de segurança, tanto na época de verão quanto no inverno. Além disso, pode-se dizer que o melhoramento para tolerância ao calor pode ser realizado fazendo-se duas avaliações por ano, utilizando cada época de semea- dura para a realização de seleção. A seleção somente em ambientes desfavoráveis não é indicada, uma vez que pode levar à obtenção de linhagens com baixo potencial produtivo (Calhoun et al., 1994).

As combinações específicas refletem o grau de complementaridade alélica entre dois genitores. Interessam ao melhorista combinações híbridas com alto valor positivo da CEC, e que pelo menos um dos genitores possua alta CGC. Neste sentido, podem ser ressaltados os cruzamentos CPAC 9186/ EP 93541, Anahuac/BH 1146 e BR 26/EP 93541, cujos genitores possuem alta CGC em todos os ambientes. Considerando somente a época de semeadura de verão, ou seja, na presença de calor, podem ser ressaltados os cruzamentos Anahuac/BR 24, BR 12/ BR 24, EMBRAPA 22/BH 1146 e EP 9320/BR 24 (Tabela 6).

Os índices de estresses de calor (\%R), determinados pela porcentagem de redução da produtividade de grãos no verão em relação ao inverno, são apresentados na Tabelas 7 e 8 . Constataram-se diferenças de tolerância ao estresse de calor entre os genitores e entre as populações híbridas, o que demonstra a existência de variabilidade genética rela-

Tabela 5. Estimativas da capacidade geral de combinação dos genitores de trigo, para o caráter produção de grãos ${ }^{(1)}$.

\begin{tabular}{|c|c|c|c|c|c|c|c|c|}
\hline \multirow[t]{3}{*}{ Genitores } & \multicolumn{4}{|c|}{ Geração $F_{1}(\mathrm{~g} / 20$ plantas $)$} & \multicolumn{4}{|c|}{ Geração $F_{2}\left(\mathrm{~g} / 4,8 \mathrm{~m}^{2}\right)$} \\
\hline & \multicolumn{2}{|c|}{ Lavras } & \multicolumn{2}{|c|}{ Patos de Minas } & \multicolumn{2}{|c|}{ Lavras } & \multicolumn{2}{|c|}{ Patos de Minas } \\
\hline & Verão & Inverno & Verão & Inverno & Verão & Inverno & Verão & Inverno \\
\hline & \multicolumn{8}{|c|}{ Grupo I } \\
\hline Anahuac & 30,55 & 3,40 & 20,56 & $-12,15$ & 79,68 & $-84,83$ & 135,32 & $-11,10$ \\
\hline BR 12 & 2,53 & $-44,20$ & $-2,16$ & $-26,51$ & 21,77 & $-30,46$ & $-43,07$ & $-39,90$ \\
\hline BR 26 & 5,05 & $-23,20$ & $-13,06$ & 15,37 & $-77,42$ & 56,67 & 108,92 & 133,30 \\
\hline CPAC 9186 & 35,69 & 22,80 & 51,74 & 63,21 & 23,45 & $-21,30$ & 3,72 & 66,10 \\
\hline EMBRAPA 22 & 7,99 & $-14,20$ & $-17,28$ & 12,47 & $-25,77$ & $-0,44$ & $-117,07$ & 5,90 \\
\hline EP 9320 & $-29,66$ & $-16,20$ & $-4,96$ & 19,51 & 74,68 & 65,29 & 93,72 & 128,90 \\
\hline IAC 24 & $-17,56$ & 39,20 & 6,14 & $-22,35$ & $-79,47$ & $-11,09$ & 40,72 & $-175,30$ \\
\hline IVI 931036 & $-34,60$ & 32,40 & $-41,00$ & $-49,57$ & $-16,92$ & 26,15 & $-222,27$ & $-107,90$ \\
\hline \multirow[t]{2}{*}{ Erro-padrão } & \multicolumn{2}{|c|}{$\mathrm{EP}\left(\hat{g}_{i}\right)=16,64$} & \multicolumn{2}{|c|}{$\mathrm{EP}\left(\hat{g}_{i}-\hat{g}_{i},\right)=25,15$} & \multicolumn{2}{|c|}{$\mathrm{EP}\left(\hat{g}_{i}\right)=41,51$} & \multicolumn{2}{|c|}{$\mathrm{EP}\left(\hat{g}_{i}-\hat{g}_{i},\right)=62,76$} \\
\hline & \multicolumn{8}{|c|}{ Grupo II } \\
\hline BH 1146 & 4,26 & 46,17 & 6,29 & $-8,30$ & 82,84 & $-114,03$ & 84,72 & $-117,20$ \\
\hline BR 24 & $-4,15$ & $-66,82$ & 1,43 & 9,69 & 49,56 & 36,13 & 152,22 & 121,17 \\
\hline EMBRAPA 21 & 3,89 & $-41,32$ & 9,97 & $-48,85$ & $-131,40$ & $-141,20$ & $-215,52$ & $-101,70$ \\
\hline EP 9287 & $-1,40$ & 21,17 & $-14,44$ & 27,39 & $-57,81$ & $-7,58$ & $-185,27$ & $-88,32$ \\
\hline EP 93541 & $-2,60$ & 40,80 & $-3,24$ & 20,07 & 56,81 & 226,70 & 163,85 & 186,05 \\
\hline Erro-padrão & \multicolumn{2}{|c|}{$\mathrm{EP}\left(\hat{g}_{j}\right)=12,58$} & $\mathrm{EP}\left(\hat{g}_{j}\right.$ & )$=19,88$ & $\mathrm{EP}(\hat{g}$ & $=31,38$ & $\mathrm{EP}\left(\hat{g}_{j}\right.$ & , $)=49,62$ \\
\hline
\end{tabular}

(1)Repetibilidade da CGC: a) pais do grupo I dentro de $\mathrm{F}_{1}=63,11$; dentro de $\mathrm{F}_{2}=52,3$; média do grupo $\mathrm{I}=60,7$; b) pais do grupo II dentro de $\mathrm{F}_{1}=11,0$; dentro de $\mathrm{F}_{2}=88,1$; média do grupo $\mathrm{II}=88,0$. 
tiva a este caráter, o que está de acordo com resultados de outros trabalhos com a cultura do trigo (Blum \& Sinmena, 1994; Wardlaw, 1994; Shukla et al., 1997). A correlação foi alta e positiva $(r=0,73$, $\mathrm{p} \leq 0,004)$ entre os índices de estresses dos parentais nos locais, mas nas populações a correlação foi baixa $(\mathrm{r}=0,38, \mathrm{p} \leq 0,016)$, apesar de significativa.

$\mathrm{Na}$ interpretação dos índices de estresses ficou evidente a sua limitação, visto que em algumas situações, como no genitor BR 12, em Lavras, e
IAC 24, em Patos de Minas (Tabela 7), baixos valores de \%R estavam associados a baixas produtividades de grãos nos dois ambientes. Diante desta dificuldade optou-se por dar maior ênfase às produtividades obtidas no verão. Entre os parentais com maior produtividade, principalmente no verão, e com baixo valor do índice de estresse, podem ser destacados: Anahuac, BH 1146, BR 24, CPAC 9186 e EP 93541. Entre as populações híbridas, podem ser salientados os cruzamentos Anahuac/BR 24,

Tabela 6. Maiores e menores valores das estimativas da capacidade específica de combinação (CEC) obtidos no cruzamento dialelo relativo ao caráter produção de grãos de trigo ${ }^{(1)}$

\begin{tabular}{|c|c|c|c|c|c|c|c|c|}
\hline \multirow[t]{3}{*}{ Cruzamentos } & \multicolumn{4}{|c|}{ Geração $F_{1}$} & \multicolumn{4}{|c|}{ Geração $F_{2}$} \\
\hline & \multicolumn{2}{|c|}{ Lavras } & \multicolumn{2}{|c|}{ Patos de Minas } & \multicolumn{2}{|c|}{ Lavras } & \multicolumn{2}{|c|}{ Patos de Minas } \\
\hline & Verão & Inverno & Verão & Inverno & Verão & Inverno & Verão & Inverno \\
\hline & \multicolumn{8}{|c|}{ Melhores CEC } \\
\hline Anahuac/BH 1146 & $-26,94$ & $-15,77$ & $-52,91$ & 46,98 & 119,11 & 66,71 & 57,67 & 140,60 \\
\hline Anahuac/BR 24 & 61,97 & $-21,77$ & 31,25 & $-23,01$ & 0,27 & 26,87 & $-15,82$ & 142,22 \\
\hline BR 12/BR 24 & 13,29 & 13,82 & 47,37 & 11,94 & $-5,04$ & 93,09 & 119,57 & $-86,97$ \\
\hline BR 26/EP 93541 & 25,52 & 128,20 & 59,04 & 34,79 & 16,82 & 315,60 & $-12,05$ & 10,95 \\
\hline CPAC 9186/EP 93541 & 7,88 & 57,20 & 19,34 & 82,15 & 113,68 & 90,08 & 57,15 & $-14,85$ \\
\hline EMBRAPA 22/BH 1146 & $-6,88$ & 24,82 & 28,23 & 14,26 & 219,82 & $-110,51$ & 153,07 & $-130,40$ \\
\hline \multirow[t]{2}{*}{ EP 9320/BR 24} & 36,89 & 96,82 & 33,67 & $-8,87$ & 132,61 & 8,75 & $-65,22$ & $-97,77$ \\
\hline & \multicolumn{8}{|c|}{ Piores CEC } \\
\hline Anahuac/EMBRAPA 21 & $-49,36$ & $-109,27$ & 33,61 & $-60,76$ & $-149,98$ & $-47,53$ & $-73,07$ & $-154,90$ \\
\hline BR 12/BH 1146 & $-1,72$ & $-17,17$ & $-14,69$ & 9.04 & $-61,12$ & $-165,59$ & $-65,92$ & 132,40 \\
\hline BR 26/EP 9287 & $-15,78$ & $-28,17$ & $-10,95$ & $-3,43$ & $-3,29$ & $-102,84$ & $-103,92$ & $-156,67$ \\
\hline EMBRAPA/22/EP 93541 & 45,48 & $-92,80$ & 6,46 & 24,19 & $-45,32$ & $-109,81$ & $-16,05$ & $-64,65$ \\
\hline EP 9320/BH 1146 & 14,98 & 0,82 & 33,21 & 40,22 & $-74,68$ & $-188,81$ & $-40,72$ & $-56,40$ \\
\hline IAC 24/EP 93541 & 3,84 & 28,80 & $-21,15$ & $-15,49$ & $-4,33$ & $-106,53$ & $-76,85$ & $-12,45$ \\
\hline
\end{tabular}

(1)Erro-padrão da geração $\mathrm{F}_{1}$ : EP $\left(\hat{s}_{i j}\right)=33,27$; EP $\left(\hat{s}_{i j}-\hat{s}_{i k}\right)=52,61 ; \operatorname{EP}\left(\hat{s}_{i j}-\hat{s}_{k j}\right)=50,30$; EP $\left(\hat{s}_{i j}-\hat{s}_{k l}\right)=46$,21; erro-padrão da geração $\mathrm{F}_{2}: \operatorname{EP}\left(\hat{s}_{i j}\right)=83,03 ; \operatorname{EP}\left(\hat{s}_{i j}-\hat{s}_{i k}\right)=131,28 ; \operatorname{EP}\left(\hat{s}_{i j}-\hat{s}_{k j}\right)=125,52 ; \operatorname{EP}\left(\hat{s}_{i j}-\hat{s}_{k l}\right)=115,30$.

Tabela 7. Classificação (Cls.) dos porcentuais de redução $(\% \mathrm{R})$ da produtividade média de grãos $\left(\mathrm{g} / 4,8 \mathrm{~m}^{2}\right)$ dos genitores de trigo, na condição de verão em relação ao inverno ${ }^{(1)}$.

\begin{tabular}{|c|c|c|c|c|c|c|c|c|}
\hline \multirow[t]{2}{*}{ Genitores } & \multicolumn{4}{|c|}{ Lavras } & \multicolumn{4}{|c|}{ Patos de Minas } \\
\hline & Verão & Inverno & $\% \mathrm{R}$ & Cls. & Verão & Inverno & $\% \mathrm{R}$ & Cls. \\
\hline Anahuac & $1.092 \mathrm{~B}$ & $2.502 \mathrm{~A}$ & 56,35 & 7 & $720 \mathrm{C}$ & $1.670 \mathrm{~B}$ & 56,89 & 7 \\
\hline BR 12 & $941 \mathrm{C}$ & $1.991 \mathrm{~B}$ & 52,71 & 5 & 541D & $1.563 \mathrm{~B}$ & 65,39 & 11 \\
\hline BR 26 & $850 \mathrm{C}$ & $2.223 \mathrm{~A}$ & 61,77 & 10 & $852 \mathrm{C}$ & $2.087 \mathrm{~A}$ & 59,18 & 8 \\
\hline CPAC 9186 & $1.368 \mathrm{~A}$ & $2.343 \mathrm{~A}$ & 41,62 & 2 & $1.277 \mathrm{~B}$ & $2.005 \mathrm{~A}$ & 36,31 & 2 \\
\hline EMBRAPA 22 & $1.007 \mathrm{C}$ & $2.267 \mathrm{~A}$ & 55,57 & 6 & $673 C$ & $1.930 \mathrm{~A}$ & 65,13 & 10 \\
\hline EP 9320 & $766 \mathrm{C}$ & $2.299 \mathrm{~A}$ & 66,68 & 13 & 579D & $2.048 \mathrm{~A}$ & 71,73 & 13 \\
\hline IAC 24 & $748 \mathrm{C}$ & $1.933 \mathrm{~B}$ & 61,28 & 8 & $844 \mathrm{C}$ & $1.628 \mathrm{~B}$ & 48,16 & 4 \\
\hline IVI 931036 & $776 \mathrm{C}$ & $2.256 \mathrm{~A}$ & 65,61 & 12 & $526 \mathrm{D}$ & $1.802 \mathrm{~B}$ & 70,81 & 12 \\
\hline BH 1146 & 1.167B & $1.936 \mathrm{~B}$ & 39,72 & 1 & $1.105 \mathrm{~B}$ & $1.680 \mathrm{~A}$ & 34,23 & 1 \\
\hline BR 24 & $1.214 \mathrm{~B}$ & $2.288 \mathrm{~A}$ & 46,95 & 3 & $1.480 \mathrm{~A}$ & $2.328 \mathrm{~A}$ & 36,43 & 3 \\
\hline EMBRAPA 21 & 897C & $2.456 \mathrm{~A}$ & 63,48 & 11 & $695 \mathrm{C}$ & $1.583 \mathrm{~B}$ & 56,10 & 6 \\
\hline EP 9287 & 889C & $2.310 \mathrm{~A}$ & 61,50 & 9 & $676 \mathrm{C}$ & $1.872 \mathrm{~B}$ & 63,89 & 9 \\
\hline EP 93541 & $1.082 \mathrm{~B}$ & $2.283 \mathrm{~A}$ & 52,58 & 4 & $1.111 \mathrm{~B}$ & $2.262 \mathrm{~A}$ & 50,88 & 5 \\
\hline Média & 984 & 2.237 & 55,83 & & 852 & 1.881 & 55,00 & \\
\hline
\end{tabular}

(1)Médias na mesma coluna seguidas pela mesma letra não se diferenciam a 5\% de probabilidade, pelo teste de Scott-Knott; erro-padrão da estimativa de $\%$ R: Lavras $=4,05$ e Patos de Minas $=4,44$. 
Tabela 8. Produtividades médias de grãos $\left(\mathrm{g} / 4,8 \mathrm{~m}^{2}\right)$ e índices de redução $(\% \mathrm{R})$ com a correspondente classificação (Cls.) das populações $F_{2}$ de trigo com os melhores e piores desempenhos ${ }^{(1)}$.

\begin{tabular}{|c|c|c|c|c|c|c|c|c|}
\hline \multirow[t]{2}{*}{ Cruzamentos } & \multicolumn{4}{|c|}{ Lavras } & \multicolumn{4}{|c|}{ Patos de Minas } \\
\hline & Verão & Inverno & $\% \mathrm{R}$ & Cls. & Verão & Inverno & $\% \mathrm{R}$ & Cls. \\
\hline & \multicolumn{8}{|c|}{ Melhores índices de desempenho } \\
\hline Anahuac/BH 1146 & $1.314 \mathrm{~A}$ & $2.176 \mathrm{~B}$ & 39,6 & 2 & $1.348 \mathrm{~A}$ & $2.152 \mathrm{~B}$ & 37,4 & 3 \\
\hline Anahuac/BR 24 & $1.162 \mathrm{~A}$ & $2.286 \mathrm{~B}$ & 49,2 & 7 & $1.342 \mathrm{~A}$ & $2.392 \mathrm{~A}$ & 43,9 & 12 \\
\hline Anahuac/EP 93541 & $1.161 \mathrm{~A}$ & $2.216 \mathrm{~B}$ & 47,6 & 6 & $1.360 \mathrm{~A}$ & $2.357 \mathrm{~A}$ & 42,3 & 5 \\
\hline CPAC 9186/BR 24 & $1.065 \mathrm{~B}$ & $2.172 B$ & 51,0 & 8 & $1.403 \mathrm{~A}$ & $2.432 \mathrm{~A}$ & 42,3 & 6 \\
\hline CPAC 9186/EP 93541 & $1.226 \mathrm{~A}$ & $2.604 \mathrm{~A}$ & 52,9 & 11 & $1.295 \mathrm{~A}$ & $2.377 \mathrm{~A}$ & 45,5 & 17 \\
\hline EP 9320/BR 24 & $1.289 \mathrm{~A}$ & $2.418 \mathrm{~A}$ & 46,7 & 5 & $1.251 \mathrm{~A}$ & $2.292 \mathrm{~A}$ & 45,4 & 16 \\
\hline \multirow{2}{*}{ EP 9320/EP 93541} & $1.169 \mathrm{~A}$ & $2.795 \mathrm{~A}$ & 58,2 & 27 & $1.440 \mathrm{~A}$ & $2.547 \mathrm{~A}$ & 43,5 & 9 \\
\hline & \multicolumn{8}{|c|}{ Piores índices de desempenho } \\
\hline BR 26/EMBRAPA 21 & $713 \mathrm{C}$ & $1.953 \mathrm{~B}$ & 63,5 & 39 & $1.211 \mathrm{~A}$ & $2.207 \mathrm{~A}$ & 45,1 & 15 \\
\hline BR 26/EP 9287 & $894 \mathrm{C}$ & $2.254 \mathrm{~B}$ & 60,3 & 34 & $890 \mathrm{C}$ & $2.028 \mathrm{~B}$ & 56,1 & 32 \\
\hline CPAC 9186/EMBRAPA 21 & $895 \mathrm{C}$ & $2.058 \mathrm{~B}$ & 56,5 & 23 & $714 \mathrm{D}$ & $2.065 \mathrm{~B}$ & 65,4 & 35 \\
\hline EMBRAPA 22/EMBRAPA 21 & $886 \mathrm{C}$ & $2.423 \mathrm{~A}$ & 63,4 & 38 & $669 \mathrm{D}$ & $2.033 \mathrm{~B}$ & 67,1 & 38 \\
\hline EMBRAPA 22/EP 9287 & $866 \mathrm{C}$ & $2.303 \mathrm{~A}$ & 62,4 & 36 & 737D & $2.188 \mathrm{~A}$ & 66,3 & 37 \\
\hline IAC 24/EMBRAPA 21 & $815 \mathrm{C}$ & $2.182 B$ & 62,6 & 37 & $1.000 \mathrm{C}$ & $1.993 \mathrm{~B}$ & 49,8 & 24 \\
\hline IVI 931036/EMBRAPA 21 & $1.066 \mathrm{~B}$ & $2.232 \mathrm{~B}$ & 52,2 & 10 & 634D & $1.945 \mathrm{~B}$ & 67,4 & 39 \\
\hline IVI 931036/EP 9287 & $965 \mathrm{C}$ & $2.350 \mathrm{~A}$ & 58,9 & 31 & $706 \mathrm{D}$ & $2.057 \mathrm{~B}$ & 65,7 & 36 \\
\hline
\end{tabular}

${ }^{(1)}$ Médias na mesma coluna seguidas pela mesma letra não se diferenciam a 5\% de probabilidade, pelo teste de Scott-Knott; erro-padrão da estimativa de $\%$ R: Lavras $=4,0$ e Patos de Minas $=4,4$.

Anahuac/BH 1146, Anahuac/EP 93541, CPAC 9186/ BR 24, CPAC 9186/EP 93541, EP 9320/BR 24 e EP 9320/EP 93541. Chama a atenção o fato de que em todas as populações destacadas, um ou dois parentais possuem boa tolerância ao calor e estão associados à boa capacidade geral de combinação.

\section{Conclusões}

1. Os efeitos aditivos e não-aditivos são importantes no controle genético da produção de grãos, na presença e na ausência de estresse de calor.

2. Os genitores Anahuac, BH 1146, BR 24, CPAC 9186, EP 9320 e EP 93541 apresentam as maiores estimativas da capacidade geral de combinação sob condições de calor.

3. Os parentais utilizados diferem quanto à tolerância ao calor, destacando-se Anahuac, BH 1146, BR 24, CPAC 9186 e EP 93541.

4. As populações híbridas Anahuac/BH 1146, Anahuac/BR 24, Anahuac/EP 93541, CPAC 9186/ BR 24, CPAC 9186/EP 93541, EP 9320/BR 24 e EP 9320/EP 93541 apresentam maior grau de tolerância ao estresse de calor.

5. As estimativas da repetibilidade dos efeitos da capacidade geral de combinação permitem inferir a possibilidade de acelerar os programas de melhora- mento conduzidos na região central do Brasil, efetuando-se seleção tanto no verão quanto no inverno.

6. A utilização de sementes na geração $F_{1}$ deve ser evitada em avaliações quanto à tolerância ao calor no campo.

\section{Referências}

ACEVEDO, E.; NACHIT, M.; FERRARA, G. O. Effects of heat stress on wheat and possible selection tools for use in breeding for tolerance. In: WHEAT FOR THE NONTRADITIONAL WARMAREAS INTERNATIONAL CONFERENCE, 1990, Foz do Iguaçu. Proceedings... Mexico: Cimmyt, 1991. p. 401-421.

BLUM, A.; SINMENA, B. Wheat seed endosperm utilization under heat stress and its relation to thermotolerance in the autotrophic plant. Field Crops Research, Amsterdam, v. 37, n. 3, p. 185-191, 1994.

BORGHI, B.; PRENZIN, M.; NASH, R. J. Combining ability estimates in bread wheat and performance of $100 \mathrm{~F}_{1}$ hybrids produced using a chemical hybridizing agent. Journal of Genetics \& Breeding, Rome, v. 43, n. 1, p. 1116, Jan. 1989.

CALHOUN, D. S.; GEBEYEHU, G.; MIRANDA, A.; RAJARAM, S.; GINKEL, M. van. Choosing evaluation environments to increase wheat grain yield under drought 
conditions. Crop Science, Madison, v. 34, n. 3, p. $673-$ 678, May/June 1994.

COMISSÃO CENTRO-BRASILEIRA DE PESQUISA DE TRIGO (Uberaba, MG). Recomendações da Comissão Centro-Brasileira de Pesquisa de Trigo para os anos de 1999/2000. Belo Horizonte: Epamig, 1999. 74 p. (Documentos, 33).

CREGAN, P. B.; BUSCH, R. H. Heterosis, inbreeding, and line performance in crosses of adapted spring wheat. Crop Science, Madison, v. 18, n. 2, p. 247-251, Mar./Apr. 1978.

CRUZ, C. D.; REGAZZI, A. J. Modelos biométricos aplicados ao melhoramento genético. Viçosa, MG: UFV, 1994. $390 \mathrm{p}$.

FOKAR, M.; NGUYEN, H. T.; BLUM, A. Heat tolerance in spring wheat: I. Estimating cellular thermo-tolerance and its heritability. Euphytica, Dordrecht, v. 104, n. 1, p. 1-8, 1998.

GRIFFING, B. Concept of general and specific combining ability in relation to diallel crossing systems. Australian Journal of Biological Sciences, Melbourne, v. 9, p. 463493, 1956.

MOFFATT, J. M.; SEARS, R. G.; COX, T. S.; PAULSEN, G. M. Wheat high temperature tolerance during reproductive growth: II. Genetic analysis of chlorophyll fluorescence. Crop Science, Madison, v. 30, n. 4, p. 886889, July/Aug. 1990.
PORTER, D. R.; NGUYEN, H. T.; BURKE, J. J. Genetic control of acquired high temperature tolerance in winter wheat. Euphytica, Dordrecht, v. 83, n. 2, p. 153-157, 1995.

SHPILER, L.; BLUM, A. Differential reaction of wheat cultivars to hot environments. Euphytica, Dordrecht, v. 35, n. 2, p. 483-492, June 1986.

SHUKLA, D. S.; GUPTA, N. K.; KAPASHI, S. B.; DESHMUKH, P. S.; SAIRAM, R. K.; PANDE, P. C. Relationship between stem reserves and grain development in terminal heat stress susceptible and tolerant wheat genotypes. Indian Journal Plant Physiology, New Delhi, v. 2, n. 1, p. 36-40, Jan./Mar. 1997.

SOUZA, E. A. Controle genético de alguns caracteres do feijoeiro. Lavras: Ufla, 1993. 105 p. Dissertação de Mestrado.

WARDLAW, I. F. The effect of high temperature on kernel development in wheat: variability related to pre-heading and pos-anthesis conditions. Australian Journal of Plant Physiology, Collingwood, v. 21, n. 4, p. 731-739, 1994.

WARDLAW, I. F.; DAWSON, I. A.; MUNIBI, P. The tolerance of wheat to high temperatures during reproductive growth: II. Grain development. Australian Journal of Agricultural Research, Collingwood, v. 40, n. 1, p. 15-24, 1989.

WRICKE, G.; WEBER, W. E. Quantitative genetics and selection in plant breeding. Berlin: Gruyter, 1986. $406 \mathrm{p}$. 